Ann. Biol. anim. Bioch. Biophys., I964, 4 (I), I7-26.

\title{
INFLUENCE DE LA DUREEE D'ÉCLAIREMENT SUR LE CONTENU HYPOPHYSAIRE EN HORMONES GONADOTROPES FSH ET ICSH CHEZ LE BÉLIER
}

\author{
J. PELLETIER et R. ORTAVANT \\ avec la collaboration technique de Marie-Madeleine de Reviers, Agnès Bure et Christine Vié. \\ Laboratoire de Physiologie de la Reproduction, \\ Centre national de Recherches zootechniques, Jouy-en-Josas (Seine-et-Oise)
}

\section{SOMMAIRE}

Soixante-dix Béliers de race Ile-de-France ont été soumis à des durées quotidiennes d'éclairement reproduisant en six mois les variations normales annuelles. Les animaux qui reçoivent en fin d'expérience 8 heures d'éclairement quotidien sont dits en "jours courts" et ceux qui sont soumis à un éclairement de 16 heures sont dits en " jours longs". Les animaux sont abattus soit à la fin de la nuit (fin de la photopériode sombre) soit à la fin du jour (fin de la photopériode claire).

La concentration en hormones gonadotropes FSH et ICSH est beaucoup plus élevée dans les hypophyses des Béliers " jours courts " que dans celles des Béliers " jours longs ".

En outre, les teneurs hypophysaires en hormones gonadotropes se situent à un niveau plus élevé au terme de la photopériode sombre qu'à la fin de la photopériode claire aussi bien chez les Béliers " jours courts " que chez les Béliers " jours longs ".

Enfin, un éclairement continu de 48 heures produit une baisse des concentrations hypophysaires de l'ordre de $85 \%$ pour FSH et $53 \%$ pour ICSH chez des Béliers "jours courts ". A titre d'hypothèse de travail, il est supposé que la lumière agit comme agent stimulant la libération des hormones gonadotropes hypophysaires mais son action prolongée bloque la synthèse hormonale qui est favorisée par l'obscurité.

Il est classiquement admis que l'activité sexuelle chez la Brebis a lieu en jours décroissants (Marshali, I937 ; Yeates, I947-I949; Hafez, I950 ; HaRT, I950). De même l'étude de l'influence de la durée de la photopériode journalière sur la spermatogenèse chez le Bélier (race Ile-de-France) a abouti à des conclusions identiques (OrTAVANT et ThibaulT, I956; OrTaVANT, I956-I96I). Il a été constaté que des Béliers soumis à une diminution graduelle de la durée quotidienne d'éclairement de I 6 heures à 8 heures présentent alors une activité spermatogénétique supérieure à celle 
d'animaux soumis à une illumination croissant de 8 heures à I 6 heures. Cette activité se manifeste par un poids testiculaire, un nombre de cellules germinales et des réserves spermatiques épididymaires bien plus élevés dans le premier cas que dans le second.

On peut penser que ces différences d'activité sexuelle sont liées à des différences d'activité hypophysaire. On sait en effet que la lumière agit sur la reproduction des Mammifères ou des Oiseaux par l'intermédiaire de l'hypophyse (BISSONNETTE, I932 ; Hill et PARKes, I933 ; Benoît, I937 et suiv. ; Donovan et Harris, I956). Cependant les études portant sur les Ovins ont abouti jusqu'à présent à des résultats contradictoires. Si certains auteurs concluent qu'il n'existe aucune ou peu de différence dans le contenu gonadotrope hypophysaire entre la saison d'anœstrus et la saison sexuelle (COLE et MILLER, I935; WARWICK, I946 ; LAMOND et al., I959; HuTchinson et RoBERTSON, I960-I962), d'autres constatent soit une légère augmentation de ce contenu au début de la saison sexuelle (REASIDE et LAMOND, I956 ; Cl,EGG et Ganong, I960) soit une diminution (KAMmlade et al., I952). Enfin AlLEN et LAmming (I 960) trouvent que chez des brebis placées en jours courts, le contenu gonadotrope de 1'hypophyse diminue par rapport à celui des brebis en anœstrus.

Nous pensons que ces contradictions proviennent soit de l'utilisation de tests insuffisamment précis ou sensibles ne permettant pas de différencier l'activité FSH de l'activité $L H$, soit du fait que peu d'auteurs ont opéré en anœstrus véritable en jours croissants, soit enfin parce que les prélèvements des hypophyses des brebis en saison sexuelle ont été effectués à des moments variables du cycle oestrien. Les réponses gonadiques du Bélier aux variations photo-périodiques étant faciles à analyser et répétables, permettent d'aborder le problème de l'action de la durée d'éclairement et du rôle des photopériodes claires et sombres sur l'activité et le contenu hypophysaires dans des conditions plus favorables.

\section{MATÉRIEL ET MÉTHODES}

Soixante-dix Béliers adultes appartenant à la race Ile-de-France ont été utilisés et mis dans des cases conditionnées photopériodiquement ainsi qu'il a été déjà écrit (ORTAVANT, 1958).

I) Les régimes lumineux artificiels: Ces régimes ont pour but de reproduire artificiellement en six mois les variations d'éclairement normalement annuelles. Dans ces expériences, les limites extrêmes des durées quotidiennes d'éclairement ont été égales à 8 heures et 16 heures (ORTAvant et Thibault, I956).

Quatre séries d'animaux ont été étudiées : trois après un cycle lumineux et demi (Série A 196r, $\left.B_{1} 1962, C 1963\right)$ et une autre après un cycle seulement $\left(B_{2} 1962\right)$ (fig. 1 ).

Les animaux qui reçoivent en fin d'expérience 8 heures d'éclairement quotidien sont dits en " jours courts " de même ceux soumis à un éclairement de 16 heures sont dits en " jours longs ". Les animaux sont abattus soit à la fin de la nuit (fin de la photopériode sombre) soit à la fin du jour (fin de la photopériode claire).

Pour étudier l'effet de la photopériode claire, des animaux " jours courts " sont divisés en deux lots : l'un sert de témoin et est abattu en fin de photopériode sombre ; l'autre est soumis à une photopériode claire continue d'une durée de 48 heures avant l'abattage. De même les béliers " jours longs " sont scindés en deux groupes, le premier abattu en fin de photopériode sombre et le second soumis à 48 heures d'obscurité continue avant abattage.

2) Étude des gonades et glandes accessoires: Les testicules sont pesés dès l'abattage. Les comptages des spermatozoides testiculaires et épididymaires sont effectués d'après la technique décrite par l'un de nous (ORTAVANT, I958). Les vésicules séminales sont pesées fraîches ; le fructose est estimé selon la technique de MaNN (1948). 
3) Dosages de FSH et de ICSH : Les hypophyses des animaux, prélevées et pesées dès l'abattage après retrait de la posthypophyse sont plongées dans l'acétone pure redistillée (Série $A$, I96I) ou immédiatement broyées à l'aide d'un homogénéiseur dans quelques $\mathrm{ml}$ d'eau bidistillée (Śries $\mathrm{B}$ et C, 1962-1963), puis congelées et lyophilisées. Toutes les estimations ont été faites sur des mélanges d'hypophyses provenant d'animaux d'un même lot.

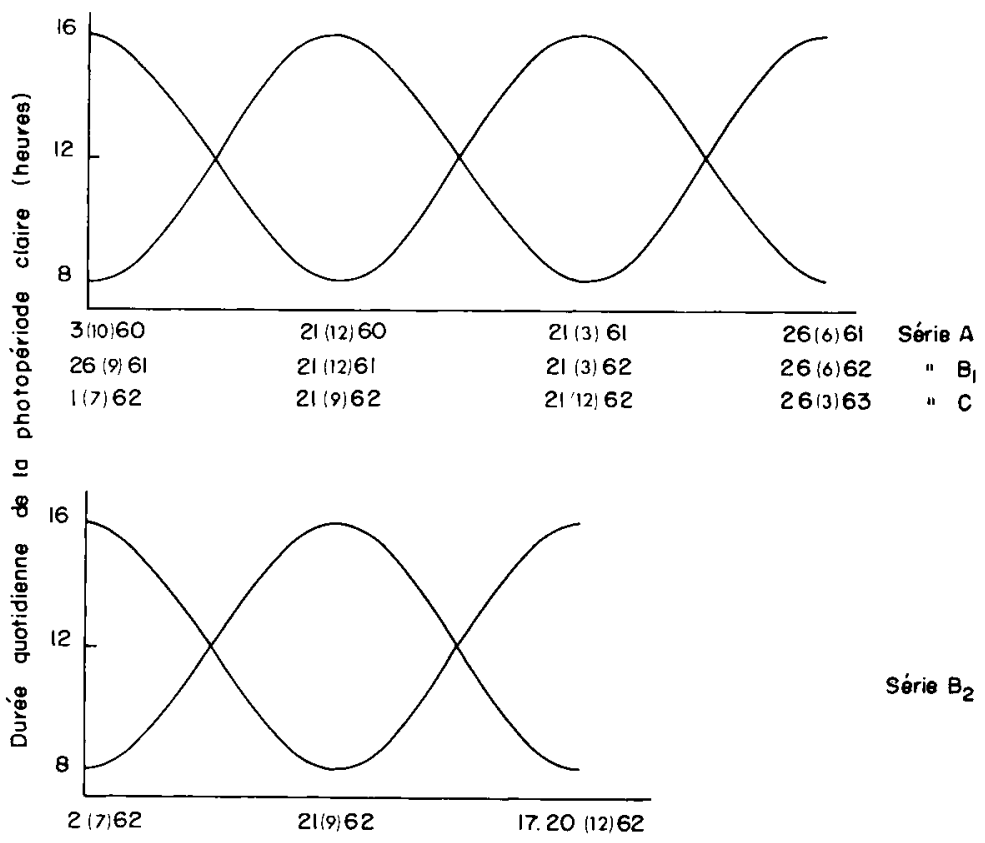

FIG. I. - Variations de la durée quolidienne d'éclairement pour les différentes séries expérimentales de Béliers

\section{a) Dosage de FSH}

Le dosage de FSH est effectué selon la technique de SteElman et Pohley (r953) sur des Rattes Wistar (souche o3, Jouy-en-Josas), âgées de 23 jours. La poudre hypophysaire acétonique ou lyophilisée est administrée par voie sous-cutanée dans du sérum physiologique avec au total 20 UI d'hormone chorionique (Produit pregnyl, Organon); chaque dose de poudre hypophysaire est testée sur 6 rattes. Pour chaque lot deux de ces doses sont comparées à deux doses de FSH-Armour. Les résultats sont exprimés en unités-équivalents du standard FSH-Armour par milligramme de poudre sèche antehypophysaire par comparaison des pentes des droites de régression (EMMENs, I948; BLISS, 1952).

\section{b) Dosage de ICSH}

La technique de Parlow (1958) modifiée par l'un de nous (Pelletier, 1963) a été appliquée à des rattes de même souche Wistar pour le dosage de ICSH. Les poudres hypophysaires sont extraites pendant 4 heures par le sérum physiologique, avec agitation magnétique. Une seule extraction étant apparue suffisante, deux doses de surnageant sont administrées par voie intraveineuse à des lots de 6 à 8 rattes parallèlement à deux doses de LH-NIH-SI qui servent de référence. L'acide ascorbique ovarien est estimé par la méthode de Mindlin et ButLer (I937). Les résultats sont exprimés en équivalent-microgrammes LH-NIH par milligramme de poudre sèche antehypophysaire par comparaison des pentes des droites de régression d'une poudre hypophysaire et du standard utilisé. Ces comparaisons sont effectuées après correction due au poids ovarien par analyse de covariance. 


\section{RÉSULTATS}

I) Poids des différentes glandes endocrines et indices d'activité physiologique

Les résultats présentés dans le tableau I indiquent que les conditions expérimentales d'éclairement quotidien conduisent à des poids testiculaires fort différents : en " jours courts" le poids du testicule est hautement significativement plus élevé $(P<o, o I)$ qu'en " jours longs " et il en est de même pour les paramètres d'activité spermatogénétique, nombre de spermatozoïdes testiculaires et réserves épididymaires. Ces données correspondent donc bien à celles qui avaient été obtenues précédemment (OrTavant et Thrbault, I956).

Au contraire, les vésicules séminales des deux groupes de béliers ne diffèrent pas pondéralement, en outre les teneurs en fructose de ces glandes sont pratiquement identiques, mais il faut reconnaître que les variations individuelles sont considérables.

Les poids des hypophyses apparaissent également semblables.

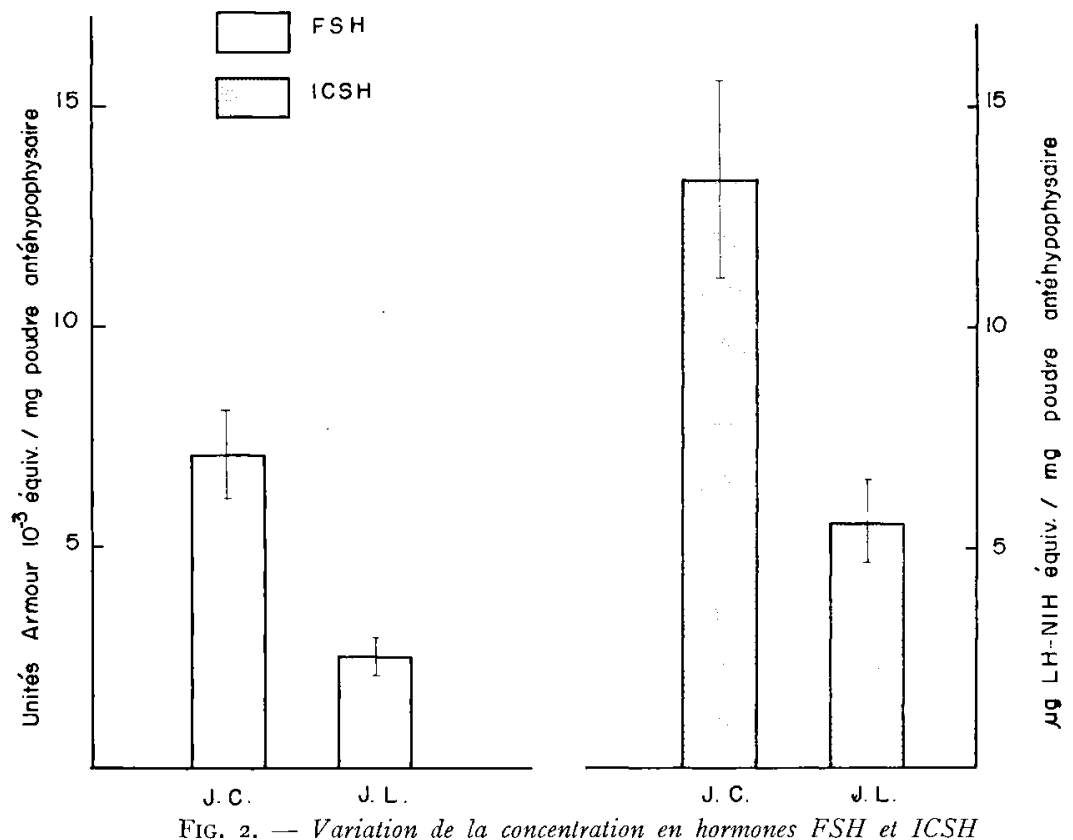

dans l'hypophyse de béliers soumis aux traitements lumineux "Jours Courts" $(J . C$.$) et "Jours Longs " (J . L$.

2) Infuence de la durée de la photopériode sur le contenu hypophysaire en FSH et ICSH

a) Les résultats (tabl. 2 et fig. 2) sont particulièrement nets. Les teneurs hypophysaires en FSH et ICSH sont hautement significativement plus élevées $(\mathrm{P}<\mathrm{o}$,or $)$ chez les animaux soumis aux photopériodes courtes : le rapport des teneurs hypophysaires " jours courts »/(jours longs » est de 2,85 en ce qui concerne FSH et 2,34 pour ICSH. 

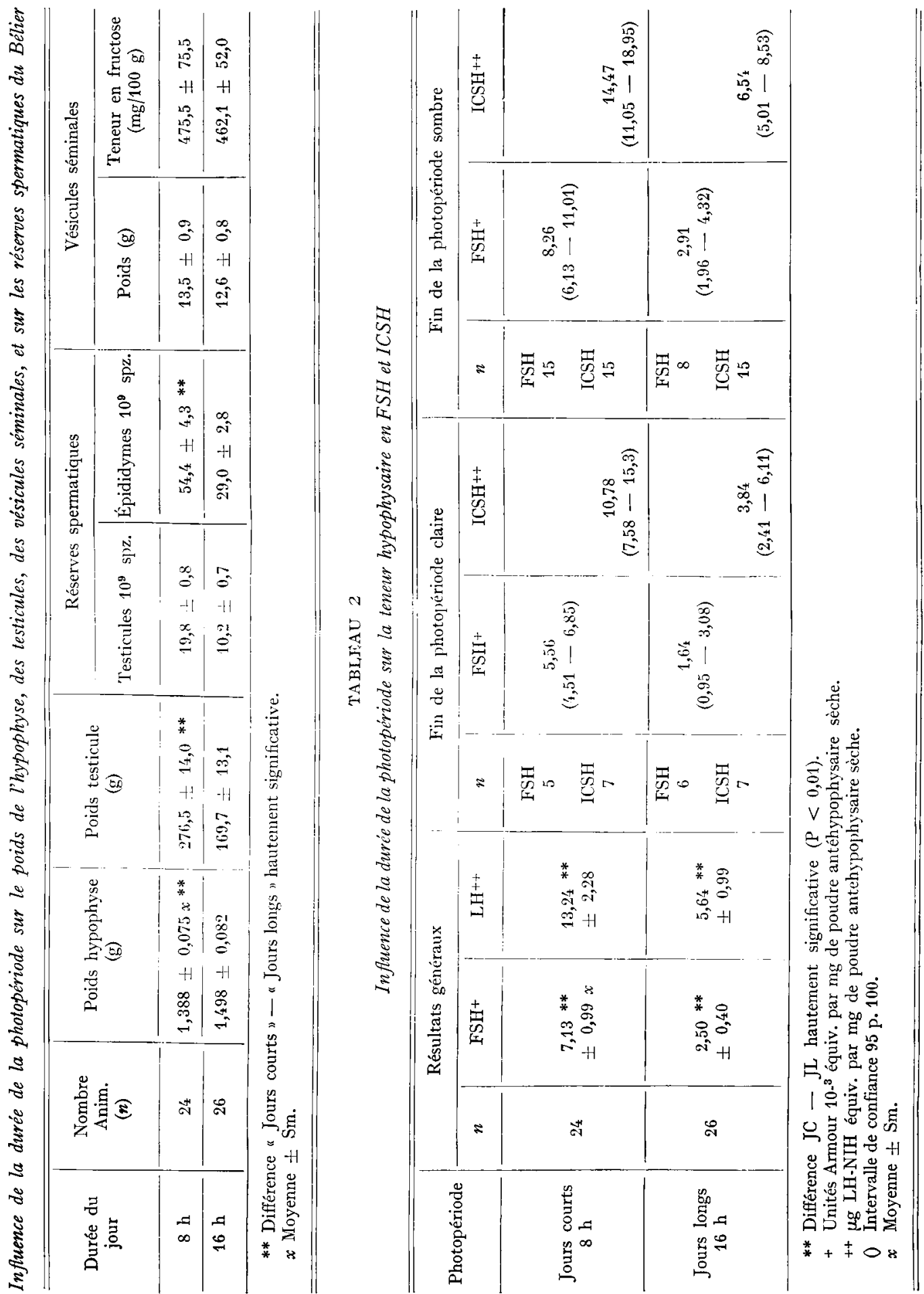


\section{TABLEAU 3}

Teneur gonadotrope hypophysaire chez le bélier soumis à différents traitements lumineux : effet des photopériodes claire et sombre

\begin{tabular}{|c|c|c|c|c|}
\hline $\begin{array}{l}\text { Fin du traitement } \\
\text { photopériodique }\end{array}$ & $\begin{array}{c}\text { Unités Armour } \\
10^{-3} \text { équiv. FSH } \\
\text { par mg de poudre } \\
\text { antehypophysaire }\end{array}$ & $\begin{array}{l}\text { Intervalle de } \\
\text { confiance } \\
(95 \%)\end{array}$ & $\begin{array}{l}\mu g \mathrm{LH}-\mathrm{NIH} \\
\text { equiv. par mg de } \\
\text { poudre antehypo- } \\
\text { physaire }\end{array}$ & $\begin{array}{l}\text { Intervalle de } \\
\text { confiance } \\
(95 \%)\end{array}$ \\
\hline $\begin{array}{l}\text { Jours courts }(8 \mathrm{~h}) \\
\text { Jours courts } \\
+\quad 48 \mathrm{~h} \text { lumière }\end{array}$ & $\left.\begin{array}{l}\text { (4) } 10,60 \\
(4) \quad 0,73\end{array}\right\} * *$ & $\begin{array}{l}8,5 / 4-13,16 \\
0,31-2,30\end{array}$ & $\begin{array}{l}\text { (4) } 23,86 \\
\text { (4) } 11,05\end{array}$ & $\begin{array}{r}11,68-48,78 \\
6,66-18,32\end{array}$ \\
\hline $\begin{array}{l}\text { Jours longs } \\
\text { Jours longs } \\
+48 \text { h obscurité }\end{array}$ & $\begin{array}{l}\text { (4) } 3,24 \\
\text { (4) } 5,0^{\prime}\end{array}$ & $\begin{array}{l}2,53-4,18 \\
3,94-6,44\end{array}$ & $\begin{array}{l}\text { (4) } 3,43 \\
\text { (4) } 4,11\end{array}$ & $\begin{array}{l}1,73-6,73 \\
2,62-6,46\end{array}$ \\
\hline
\end{tabular}

** Différence hautement significative $(\mathrm{P}<0,01)$.

() Nombre d'animaux.

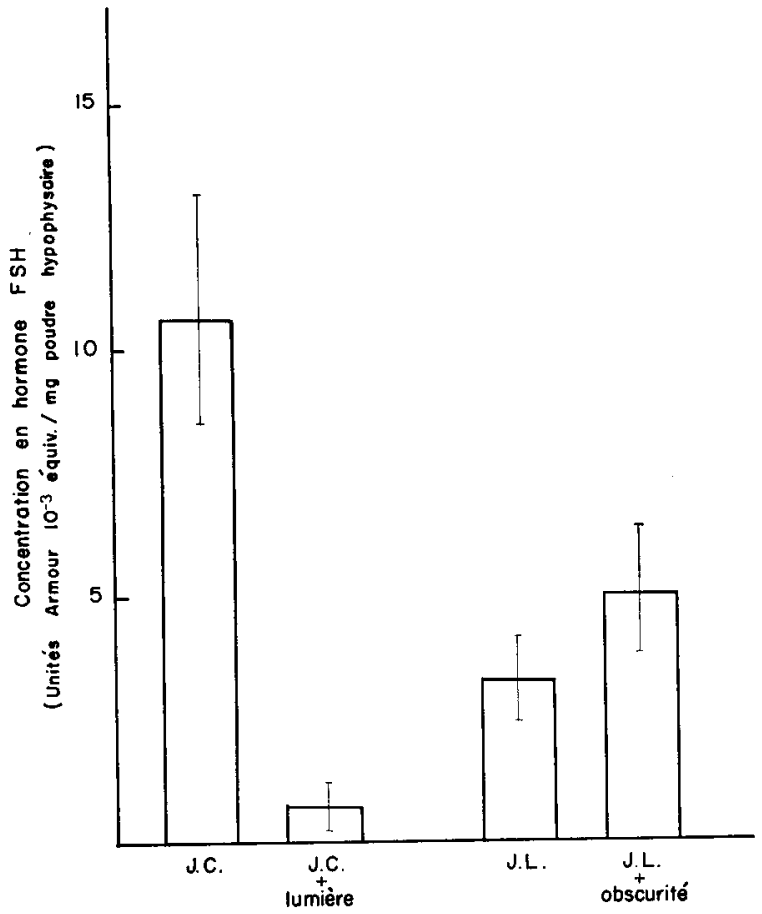

FIG. 3. - Variation de la concentration en hormone FSH dans l'hypophyse de béliers soumis d différents traitements lumineux a la fin de la photopériode sombre. (J.C.: Jours Courts; J. L. : Jours Longs, Lumière: $48 \mathrm{~h}$ d'illumination continue ; obscurité : $48 \mathrm{~h}$ d'obscurité continue; concentration \pm intervalle de confiance $95 \%$.) 
b) Mais pour un même lot " jour court " ou " jour long " le sacrifice des animaux soit à la fin de la photopériode sombre soit à la fin de la photopériode claire révèle une très remarquable différence dans les teneurs hypophysaires. A la fin de la photopériode claire, les contenus en FSH et ICSH sont notablement moins importants qu'à la fin de la photopériode sombre. Bien que ces différences ne soient significatives $(\mathrm{P}<0,05)$ que dans le cas de ICSH en " jour long ", elles se retrouvent toutefois pour les deux hormones, tant en " jour court " qu'en " jour long ".

Cette constatation laisse supposer l'existence d'un rythme nycthéméral et pose en réalité le problème très important du rôle de la lumière comme agent de stimulation de la libération des hormones.

3) Influence de la lumière et de l'obscurité continues sur les teneurs hypophysaires

a) Effet de la lumière. en FSH et ICSH

Les résultats (tabl. 3 et fig. 3-4) montrent une diminution de la teneur hypophysaire extrêmement importante sous l'action d'une période d'éclairement continu de 48 heures : 84 p. Ioo dans le cas de FSH, 53 p. Ioo dans celui de ICSH ; ces différences sont hautement significatives $(\mathrm{P}<\mathrm{O}, \mathrm{OI})$.

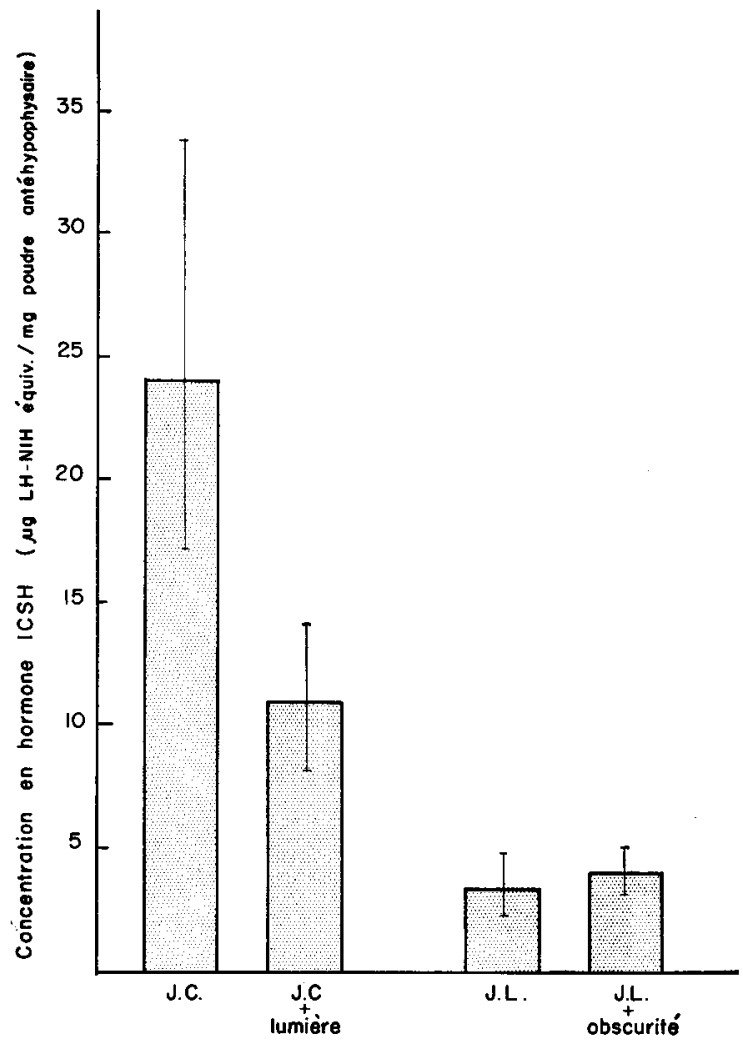

FIG. 4. - Variation de la concentration en hormone ICSH dans l'hypophyse de Béliers soumis a différents traitements lumineux à la fin de la photopériode sombre. (J.C.: Jours Courts; J. L. : Jours Longs; Lumière: 48 h d'illumination continue; obscurité : 48 h d'obscurité continue; concentration \pm erreur-lype.) 
Une expérience effectuée dans des conditions moins favorables que la précédente, les âges et les origines des animaux étant variables, a conduit cependant à un résultat du même ordre. La décroissance de FSH était de 65 p. Ioo, celle de ICSH de 59 p. Ioo, ces différences sont aussi hautement significatives.

\section{b) Mise à l'obscurité.}

En revanche la mise à l'obscurité se traduit par une faible élévation de la teneur en !hormone de l'hypophyse pour FSH $(\mathrm{P}<0,05)$, celle concernant ICSH n'étant pas significative.

\section{CONCLUSION}

Un certain nombre de résultats apparaissent particulièrement nets :

I) La différence entre les teneurs hormonales des hypophyses des béliers “ jours courts " et " jours longs " est hautement significative, cette teneur étant la plus forte chez les premiers qui manifestent par ailleurs la plus grande activité testiculaire. Ceci conduit à penser que, lors des photopériodes claires décroissantes il y aurait sécrétion et décharge accrues de FSH et ICSH. Les teneurs hypophysaires les plus élevées correspondent aux fortes activités sexuelles et inversement, il est donc vraisemblable que les résultats contradictoires des auteurs, concernant les teneurs hypophysaires chez la Brebis sont dus au choix de la période d'ancestrus qui doit être limitée aux photopériodes claires croissantes. Un problème est toutefois soulevé en ce qui concerne ICSH, puisque les vésicules séminales et leur teneur en fructose restent inchangées quelle que soit la photopériode. Il est possible que ces critères soient très peu sensibles à ICSH ou que les fortes variations individuelles observées empêchent de conclure.

2) Les résultats acquis par les abattages des animaux à la fin de la photopériode sombre ou claire semblent indiquer l'existence d'un cycle nycthéméral. Ce cycle comprendrait en " jours courts » comme en " jours longs » une charge de 1'hypophyse durant les périodes d'obscurité et la libération après une durée minimum d'éclairement.

Les données obtenues par l'action de 48 heures de lumière continue indiquent que l'excitation lumineuse entraîne une diminution considérable de la teneur hypophysaire en FSH et ICSH, qu'on peut très vraisemblablement attribuer à une décharge à partir de 1'hypophyse. La lumière serait donc chez les Ovins comme chez les Oiseaux, un agent de stimulation de la libération des hormones gonadotropes. La sécrétion pourrait être au contraire sous la dépendance de périodes d'obscurité qui devraient être d'une durée suffisante. 


\title{
SUMMARY
}

\author{
INFLUENCE OF DURATION OF LIGHT ON THE CONTENT \\ OF GONADOTROPHIC HORMONES FSH AND ICSH IN THE HYPOPHYSIS OF THE RAM
}

The progressive conditioning to light of rams exposed to 8 or 16 hours of illumination daily is shown by the fact that in the animals given short periods of light the hypophysis contains much more FSH and ICSH, correlated with greater testicular activity.

Moreover, the contents of the hormones in the hypophysis seem to be greater at the end of the period of darkness than at the end of the period of light. This applies to both FSH and ICSH during " short " and "long " days. It may be assumed that their is a diurnal cycle, with the release of hormones during the day.

The effect of light on the release of gonadotrophic hormones is shown also by giving continuous light for 48 hours, which causes a reduction of FSH in the hypophysis of the order of $85 \mathrm{p}$. I 00 and of ICSH of $53 \mathrm{p}$. IOO. As a working hypothesis it is suggested that light acts as an agent which stimulates release of hormones from the hypophysis, but its prolonged action blocks the synthesis of hormones, which is favoured by darkness.

\section{RÉFÉRENCES BIBLIOGRAPHIQUES}

Allen D. M., Lamming G. E., I960. The induction of breeding activity in lactating ewes during anostrus. J. Reprod. Fertil., 1, 21 3-222.

BENoît J., 1937. Facteurs externes et internes de l'activité sexuelle. II. Étude du mécanisme de la stimulation par la lumière de l'activité testiculaire chez le canard domestique. Rôle de l'hypophyse. Bull. Biol. Fr. el Belg., 71, 393-437.

Bissonnette J. H., 1932. Modifications of Mammalian sexual cycles : reactions of ferrets (Pulorius Vulgarls) of both sexes to electric light added after dark in November and December. Proc. Roy. Soc. B, 110, $322-336$.

BuISS C. I., I952. The statistics of Bioassay, with special reference to the vitamins. Acad. Press. Nere York, $447-628$.

Clegg M. T., GANONG W. F., I960. The effect of hypothalamic lesions on ovarian function in the ewe. Endocrinology, 67, 179-1 86.

Cole H. H., Miller R. F., I935. The changes in the reproductive organs of the ewe with some data bearing on their control. Amer. J. Anat., 5\%, 39-97.

Donovan B. T., Harris G. W., 1956. The effect of pituitary stalk section on light induced estrus in the ferret. J. Physiol., 131, I02-I I4.

Emmens C. W., I948. Principles of biological assay. Chapman et Hall. London, $206 \mathrm{p}$.

HAFEZ E. S. E., 1950. Sexual season of the ewe and daylight environment. Nature, 166, 822.

Hart D. S., I950. Periodicity in Suffolk sheeps. J. agric. Sci., 40, I43-I 49.

HiLl M., PARkes A. S., I933. Studies on the hypophysectomized ferret. V. Effect of hypophysectomy on the response of the female ferret to additional illumination during ancestrus. Proc. Roy. Soc. B, 113, 537. 540 .

Hutchinson J. S. M., Robertson H., ig60. Effect of season on the follicle stimulating hormone and luteinizing hormone potency of sheep anterior pituitary glands. Nature, 188, 585-586.

Kammlade W. G., Welch J. A., Nalbandov A. V., Norton H. N., I952. Pituitary activity of sheep in relation to the breeding season. J. anim. Sci., 11, 646-655.

LAmond D. R., Radford H. M., Wallace A. L., I959. Bioassay of sheep anterior pituitary glands Nature, 183, I 597-1598.

Mann T., I948. Fructose content and fructolysis in semen. Practical application in the evaluation of semen quality. J. agric. Sci., 38, 32 I-33I.

MaRsiall F. H. A., I937. On the change over in the oestrous cycle in animals after transference across the equator, with further observations on the incidence of the breeding seasons and the factor controlling periodicity. Proc. Roy. Soc. B, 122, 4I 3-428.

Mindlin R. L., Butler A. M., 1937. The determination of ascorbic acid in plasma. J. Biol. Chem., 122, $673-686$. 
Ortavant R., Thibault C., I956. Influence de la durée d'éclairement sur les productions spermatiques du bélier. C. R. Soc. Biol., 150, 358-36r.

Ortavant R., Thibault C., I956. Action de la durée d'éclairement sur les processus spermatogénétiques chez le bélier. C. R. Soc. Biol., 150, 47 I-474.

Ortavant R., 1958. Le cycle spermatogénétique chez le bélier. Thèse doct., Paris, p. I27 éd 1959, Ann. Zootech., 8, I83-244 et 27I-322.

Ortavant R., I96r. Réponses spermatogénétiques du bélier à différentes durées d'éclairement. $I V$ e Congr. intern. reprod. anim., La Haye, $236-242$.

Parlow A. F., 1958. A rapid bioassay method for LH and factors stimulating LH secretion. Feder. Proc., 17, 402 .

Pelletier J., 1963. Étude critique du dosage de ICSH par la méthode de l'acide ascorbique ovarien. Ann. Biol. anim. Bioch. Biophys., 3, 307-323.

RAESIDE J. I., LAMOND D. R., I956. Effects of progesterone and pregnant mare serum (PMS) administration in the anœstrous ewe. Aust. J. A Aric. Res., 7, 591-600.

Robertson H. A., Hutcinnson J. S. M., ig62. The levels of FSH and LH in the pituitary of the ewe inrelation to follicular growth and ovulation. J. Endocrin., 24, I43-15I.

Steelman S. L., Pohley R. P., 1953. Assay of the follicle stimulating hormone based on the augmentation with human chorionic gonadotrophin. Endocrinology, 53, 604-616.

WARWICK E. J., I946. Gonadotrophic potency of ewe pituitary glands as affected by spaying, season and breed. Proc. Soc. exp. Biol. N. Y., 63, 530-533.

YEATES N. T. M., 1947. Influence of variation in length day upon the breeding season in sheep. Nature, $160,429-430$.

YEaTES N. T. M., I949. The breeding season of the sheep with particular reference to its modification by artificial means using light. J. Agr. Sci., 39, $\mathrm{I}-43$. 